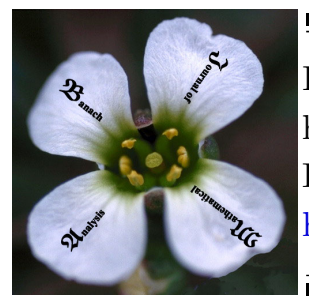

Banach J. Math. Anal. 9 (2015), no. 2, 9-20

http://doi.org/10.15352/bjma/09-2-2

ISSN: $1735-8787$ (electronic)

http://projecteuclid.org/bjma

\title{
SOME HERMITE-HADAMARD TYPE INEQUALITIES FOR THE PRODUCT OF TWO OPERATOR PREINVEX FUNCTIONS
}

\author{
A. G. GHAZANFARI* AND A. BARANI \\ Communicated by F. Kittaneh
}

\begin{abstract}
In this paper we introduce operator preinvex functions and establish a Hermite-Hadamard type inequality for such functions. We give an estimate of the right hand side of a Hermite-Hadamard type inequality in which some operator preinvex functions of selfadjoint operators in Hilbert spaces are involved. Also some Hermite-Hadamard type inequalities for the product of two operator preinvex functions are given.
\end{abstract}

\section{INTRODUCTION}

The following inequality holds for any convex function $f$ defined on $\mathbb{R}$ and $a, b \in \mathbb{R}$, with $a<b$

$$
f\left(\frac{a+b}{2}\right) \leq \frac{1}{b-a} \int_{a}^{b} f(x) d x \leq \frac{f(a)+f(b)}{2}
$$

Both inequalities hold in the reversed direction if $f$ is concave. We note that Hermite-Hadamard's inequality may be regarded as a refinement of the concept of convexity and it follows easily from Jensen's inequality. The classical HermiteHadamard inequality provides estimates of the mean value of a continuous convex function $f:[a, b] \rightarrow \mathbb{R}$. The Hermite-Hadamard inequality has several applications in nonlinear analysis and the geometry of Banach spaces, see [10, 5].

Date: Received: Feb. 16, 2014; Accepted: May 9, 2014.

* Corresponding author.

2010 Mathematics Subject Classification. Primary 47A63; Secondary 26D07, 26D15.

Key words and phrases. Hermite-Hadamard inequality, invex sets, operator preinvex functions. 
In recent years several extensions and generalizations have been considered for classical convexity. We would like to refer the reader to [3, 7, 17] and references therein for more information. A number of papers have been written on this inequality providing some inequalities analogous to Hadamard's inequality given in (1.1) involving two convex functions, see [14, 11, 16]. Pachpatte in [14] has proved the following theorem for the product of two convex functions.

Theorem 1.1. Let $f$ and $g$ be real-valued, nonnegative and convex functions on $[a, b]$. Then

$$
\begin{gathered}
\frac{1}{b-a} \int_{a}^{b} f(x) g(x) d x \leq \frac{1}{3} M(a, b)+\frac{1}{6} N(a, b), \\
2 f\left(\frac{a+b}{2}\right) g\left(\frac{a+b}{2}\right) \leq \frac{1}{b-a} \int_{a}^{b} f(x) g(x) d x+\frac{1}{6} M(a, b)+\frac{1}{3} N(a, b),
\end{gathered}
$$

where $M(a, b)=f(a) g(a)+f(b) g(b), N(a, b)=f(a) g(b)+f(b) g(a)$.

A significant generalization of convex functions is that of invex functions introduced by Hanson in [9]. In this paper we introduce operator preinvex functions and give an operator version of the Hermite-Hadamard inequality for such functions.

First, we review the operator order in $B(H)$ and the continuous functional calculus for a bounded selfadjoint operator. For selfadjoint operators $A, B \in$ $B(H)$ we write $A \leq B$ (or $B \geq A$ ) if $\langle A x, x\rangle \leq\langle B x, x\rangle$ for every vector $x \in H$, we call it the operator order.

Now, let $A$ be a bounded selfadjoint linear operator on a complex Hilbert space $(H ;\langle.,\rangle$.$) and C(S p(A))$ the $C^{*}$-algebra of all continuous complex-valued functions on the spectrum of $A$. The Gelfand map establishes a $*$-isometrically isomorphism $\Phi$ between $C(S p(A))$ and the $C^{*}$-algebra $C^{*}(A)$ generated by $A$ and the identity operator $1_{H}$ on $H$ as follows (see for instance [15, p.3]): For $f, g \in C(S p(A))$ and $\alpha, \beta \in \mathbb{C}$

(i) $\Phi(\alpha f+\beta g)=\alpha \Phi(f)+\beta \Phi(g)$;

(ii) $\Phi(f g)=\Phi(f) \Phi(g)$ and $\Phi\left(f^{*}\right)=\Phi(f)^{*}$;

(iii) $\|\Phi(f)\|=\|f\|:=\sup _{t \in S p(A)}|f(t)|$;

(iv) $\Phi\left(f_{0}\right)=1$ and $\Phi\left(f_{1}\right)=A$, where $f_{0}(t)=1$ and $f_{1}(t)=t$, for

$t \in S p(A)$.

If $f$ is a continuous complex-valued functions on $S p(A)$, the element $\Phi(f)$ of $C^{*}(A)$ is denoted by $f(A)$, and we call it the continuous functional calculus for a bounded selfadjoint operator $A$.

If $A$ is a bounded selfadjoint operator and $f$ is a real-valued continuous function on $S p(A)$, then $f(t) \geq 0$ for any $t \in S p(A)$ implies that $f(A) \geq 0$, i.e., $f(A)$ is a positive operator on $H$. Moreover, if both $f$ and $g$ are real-valued functions on $S p(A)$ such that $f(t) \leq g(t)$ for any $t \in \operatorname{sp}(A)$, then $f(A) \leq f(B)$ in the operator order in $B(H)$. 
A real valued continuous function $f$ on an interval $I$ is said to be operator convex (operator concave) if

$$
f((1-\lambda) A+\lambda B) \leq(\geq)(1-\lambda) f(A)+\lambda f(B)
$$

in the operator order in $B(H)$, for all $\lambda \in[0,1]$ and for every bounded self-adjoint operators $A$ and $B$ in $B(H)$ whose spectra are contained in $I$.

For some fundamental results on operator convex (operator concave) and operator monotone functions, see $[15,4]$ and the references therein.

Dragomir in [6] has proved a Hermite-Hadamard type inequality for operator convex functions:

Theorem 1.2. Let $f: I \rightarrow \mathbb{R}$ be an operator convex function on the interval I. Then for any selfadjoint operators $A$ and $B$ with spectra in $I$ we have the inequality

$$
\begin{aligned}
\left(f\left(\frac{A+B}{2}\right) \leq\right) \frac{1}{2}\left[f\left(\frac{3 A+B}{4}\right)+f\left(\frac{A+3 B}{4}\right)\right] \\
\left.\leq \int_{0}^{1} f((1-t) A+t B)\right) d t \\
\leq \frac{1}{2}\left[f\left(\frac{A+B}{2}\right)+\frac{f(A)+f(B)}{2}\right]\left(\leq \frac{f(A)+f(B)}{2}\right) .
\end{aligned}
$$

Moslehian in [13] generalized the above theorem 1.2 as follows:

Theorem 1.3. If $A, B$ are self-adjoint operators on a Hilbert space $H$ with spectra in an interval $J, f$ is an operator convex function on $J$ and $k, p$ are positive integers, then

$$
\begin{aligned}
f\left(\frac{A+B}{2}\right) \leq \frac{1}{k^{p}} \sum_{i=0}^{k^{p}-1} f( & \left.\frac{2 i+1}{2 k^{p}} A+\left(1-\frac{2 i+1}{2 k^{p}}\right) B\right) \\
\left.\leq \int_{0}^{1} f((1-t) A+t B)\right) d t & \\
\leq \frac{1}{2 k^{p}} \sum_{i=0}^{k^{p}-1}\left[f\left(\frac{i+1}{k^{p}} A+\left(1-\frac{i+1}{k^{p}}\right) B\right)+f\left(\frac{i}{k^{p}} A+\left(1-\frac{i}{k^{p}}\right) B\right)\right] & \leq \frac{f(A)+f(B)}{2} .
\end{aligned}
$$

Motivated by the above results we investigate in this paper the operator version of the Hermite-Hadamard inequality for operator preinvex functions. We show that Theorem 1.3 holds for operator preinvex functions and establish an estimate of the right hand side of a Hermite-Hadamard type inequality in which some operator preinvex functions of selfadjoint operators in Hilbert spaces are involved. We also give some Hermite-Hadamard type inequalities for the product of two operator preinvex functions. 


\section{OPERATOR PREINVEX FUNCTIONS}

Definition 2.1. Let $X$ be a real vector space, a set $S \subseteq X$ is said to be invex with respect to the map $\eta: S \times S \rightarrow X$, if for every $x, y \in S$ and $t \in[0,1]$,

$$
y+t \eta(x, y) \in S \text {. }
$$

It is obvious that every convex set is invex with respect to the map $\eta(x, y)=$ $x-y$, but there exist invex sets which are not convex (see [1]).

Let $S \subseteq X$ be an invex set with respect to $\eta: S \times S \rightarrow X$. For every $x, y \in S$ the $\eta$-path $P_{x v}$ joining the points $x$ and $v:=x+\eta(y, x)$ is defined as follows

$$
P_{x v}:=\{z: z=x+t \eta(y, x): t \in[0,1]\} .
$$

The mapping $\eta$ is said to be satisfies the condition $C$ if for every $x, y \in S$ and $t \in[0,1]$,

$$
\text { (C) } \quad \begin{aligned}
\eta(y, y+t \eta(x, y)) & =-t \eta(x, y), \\
\eta(x, y+t \eta(x, y)) & =(1-t) \eta(x, y) .
\end{aligned}
$$

Note that for every $x, y \in S$ and every $t_{1}, t_{2} \in[0,1]$ from condition $C$ we have

$$
\eta\left(y+t_{2} \eta(x, y), y+t_{1} \eta(x, y)\right)=\left(t_{2}-t_{1}\right) \eta(x, y),
$$

see $[12,18]$ for details.

Let $\mathcal{A}$ be a $C^{*}$-algebra, denote by $\mathcal{A}_{s a}$ the set of all self adjoint elements in $\mathcal{A}$.

Definition 2.2. Let $I$ be an interval in $\mathbb{R}$ and $S \subseteq B(H)_{s a}$ be an invex set with respect to $\eta: S \times S \rightarrow B(H)_{s a}$. A continuous function $f: I \rightarrow \mathbb{R}$ is said to be operator preinvex on $I$ with respect to $\eta$ for operators in $S$ if

$$
f(B+t \eta(A, B)) \leq(1-t) f(B)+t f(A) .
$$

in the operator order in $B(H)$, for all $t \in[0,1]$ and for every $A, B \in S$ whose spectra are contained in $I$.

Every operator convex function is an operator preinvex with respect to the map $\eta(A, B)=A-B$ but the converse does not holds (see the following example).

Now, we give an example of some operator preinvex functions and invex sets with respect to the maps $\eta$ which satisfy the conditions (C).

Example 2.3. (a) Suppose that $1_{H}$ is the identity operator on a Hilbert space $H$, and

$$
\begin{aligned}
& T:=\left\{A \in B(H)_{s a}: A \leq-1 \times 1_{H}\right\} \\
& U:=\left\{A \in B(H)_{s a}: 1_{H} \leq A\right\} \\
& S:=T \cup U \subseteq B(H)_{s a} .
\end{aligned}
$$

Suppose that the function $\eta_{1}: S \times S \rightarrow B(H)_{s a}$ is defined by

$$
\eta_{1}(A, B)= \begin{cases}A-B & A, B \in U, \\ A-B & A, B \in T, \\ 1_{H}-B & A \in T, B \in U, \\ -1_{H}-B & A \in U, B \in T .\end{cases}
$$


Clearly $\eta_{1}$ satisfies condition $C$ and $S$ is an invex set with respect to $\eta_{1}$. We show that the real function $f(t)=t^{2}$ is operator preinvex with respect to $\eta_{1}$ on every interval $I \subseteq \mathbb{R}$, for operators in $S$. Since $f$ is an operator convex function on $I$, for the cases which $\eta_{1}(A, B)=A-B$ the inequality (2.3) holds. Let $\eta_{1}(A, B)=1_{H}-B$, in this case we have $1_{H} \leq-A \leq A^{2}$ and

$$
\begin{aligned}
\left(B+t \eta_{1}(A, B)\right)^{2}= & \left(B+t\left(1_{H}-B\right)\right)^{2}=\left((1-t) B+t 1_{H}\right)^{2} \\
& \leq(1-t) B^{2}+t 1_{H} \leq(1-t) B^{2}+t A^{2} .
\end{aligned}
$$

Similarly, for the case $\eta_{1}=-1_{H}-B$ we have

$$
\begin{array}{r}
\left(B+t \eta_{1}(A, B)\right)^{2}=\left(B+t\left(-1_{H}-B\right)\right)^{2}=\left((1-t)(-B)+t 1_{H}\right)^{2} \\
\leq(1-t) B^{2}+t 1_{H} \leq(1-t) B^{2}+t A^{2}
\end{array}
$$

therefore, the inequality (2.3) holds.

But the real function $g(t)=a+b t, \quad a, b \in \mathbb{R}$ is not operator preinvex with respect to $\eta_{1}$ on $S$.

(b) Suppose that $V:=\left(-2 \times 1_{H}, 0\right), W:=\left(0,2 \times 1_{H}\right), S:=V \cup W \subseteq B(H)_{s a}$ and the function $\eta_{2}: S \times S \rightarrow B(H)_{s a}$ is defined by

$$
\eta_{2}(A, B)= \begin{cases}A-B & A, B \in V \text { or } A, B \in W, \\ 0 & \text { otherwise } .\end{cases}
$$

Clearly $\eta_{2}$ satisfies condition $C$ and $S$ is an invex set with respect to $\eta_{2}$. The constant functions $f(t)=a, a \in \mathbb{R}$ is only operator preinvex functions with respect to $\eta_{2}$ for operators in $S$. Because for $\eta_{2}=0$,

$$
f\left(B+t \eta_{2}(A, B)\right)=f(B) \leq(1-t) f(B)+t f(A),
$$

implies that $f(A)-f(B) \geq 0$. interchanging $A, B$ we get $f(B)-f(A) \geq 0$.

(c) The function $f(t)=-|t|$ is not a convex function, but it is a operator preinvex function with respect to $\eta_{3}$, where

$$
\eta_{3}(A, B)= \begin{cases}A-B & A, B \geq 0 \text { or } A, B \leq 0, \\ B-A & \text { otherwise } .\end{cases}
$$

Let $X$ be a vector space, $x, y \in X, x \neq y$. Define the segment

$$
[x, y]:=(1-t) x+t y ; t \in[0,1] .
$$

We consider the function $f:[x, y] \rightarrow \mathbb{R}$ and the associated function

$$
\begin{aligned}
& g(x, y):[0,1] \rightarrow \mathbb{R}, \\
& g(x, y)(t):=f((1-t) x+t y), t \in[0,1] .
\end{aligned}
$$

Note that $\mathrm{f}$ is convex on $[x, y]$ if and only if $g(x, y)$ is convex on $[0,1]$. For any convex function defined on a segment $[x, y] \in X$, we have the Hermite- Hadamard integral inequality

$$
f\left(\frac{x+y}{2}\right) \leq \int_{0}^{1} f((1-t) x+t y) d t \leq \frac{f(x)+f(y)}{2}
$$


which can be derived from the classical Hermite-Hadamard inequality (1.1) for the convex function $g(x, y):[0,1] \rightarrow \mathbb{R}$.

Proposition 2.4. Let $I$ be an interval in $\mathbb{R}, S \subseteq B(H)_{\text {sa }}$ an invex set with respect to $\eta: S \times S \rightarrow B(H)_{\text {sa }}$ and $\eta$ satisfies condition $C$ on $S$ and $f: I \rightarrow \mathbb{R} a$ continuous function. Then, for every $A, B \in S$ with spectra of $A, V:=A+\eta(B, A)$ in $I$, the function $f$ is operator preinvex on $I$ with respect to $\eta$ for operators in $\eta$-path $P_{A V}$ if and only if the function $\varphi_{x, A, B}:[0,1] \rightarrow \mathbb{R}$ defined by

$$
\varphi_{x, A, B}(t):=\langle f(A+\operatorname{t\eta }(B, A)) x, x\rangle
$$

is convex on $[0,1]$ for every $x \in H$ with $\|x\|=1$.

Proof. Let the function $f$ be operator preinvex on $I$ with respect to $\eta$ for operators in $\eta$-path $P_{A V}$. Suppose that $A, B \in S$ with spectra $A, V$ in $I$, since $A+$ $t \eta(B, A)=t V+(1-t) A$ therefore for all $t \in[0,1], S p(A+t \eta(B, A)) \subseteq I$. If $t_{1}, t_{2} \in[0,1]$ since $\eta$ satisfies condition $C$ on $S$, we have

$$
\begin{aligned}
\varphi_{x, A, B}\left((1-\lambda) t_{1}\right. & \left.+\lambda t_{2}\right)=\left\langle f\left(A+\left((1-\lambda) t_{1}+\lambda t_{2}\right) \eta(B, A)\right) x, x\right\rangle \\
& =\left\langle f\left(A+t_{1} \eta(B, A)+\lambda \eta\left(A+t_{2} \eta(B, A), A+t_{1} \eta(B, A)\right) x, x\right\rangle\right. \\
& \leq \lambda\left\langle f\left(A+t_{2} \eta(B, A)\right) x, x\right\rangle+(1-\lambda)\left\langle f\left(A+t_{1} \eta(B, A)\right) x, x\right\rangle \\
& =\lambda \varphi_{x, A, B}\left(t_{2}\right)+(1-\lambda) \varphi_{x, A, B}\left(t_{1}\right),
\end{aligned}
$$

for every $\lambda \in[0,1]$ and $x \in H$ with $\|x\|=1$. Therefore, $\varphi_{x, A, B}$ is convex on $[0,1]$.

Conversely, suppose that $x \in H$ with $\|x\|=1$ and $\varphi_{x, A, B}$ is convex on $[0,1]$ and $C_{1}:=A+t_{1} \eta(B, A) \in P_{A V}, C_{2}:=A+t_{2} \eta(B, A) \in P_{A V}$. Fix $\lambda \in[0,1]$. By (2.4) we have

$$
\begin{aligned}
\left\langle f\left(C_{1}+\lambda \eta\left(C_{2}, C_{1}\right)\right) x, x\right\rangle & =\left\langle f\left(A+\left((1-\lambda) t_{1}+\lambda t_{2}\right) \eta(B, A)\right) x, x\right\rangle \\
& =\varphi_{x, A, B}\left((1-\lambda) t_{1}+\lambda t_{2}\right) \\
& \leq(1-\lambda) \varphi_{x, A, B}\left(t_{1}\right)+\lambda \varphi_{x, A, B}\left(t_{2}\right) \\
& =(1-\lambda)\left\langle f\left(C_{1}\right) x, x\right\rangle+\lambda\left\langle f\left(C_{2}\right) x, x\right\rangle .
\end{aligned}
$$

Hence, $f$ is operator preinvex with respect to $\eta$ for operators in $\eta$-path $P_{A V}$.

\section{HeRmite-Hadamard type inequalities}

In this section we generalize Theorem 1.1 and Theorem 1.3 for operator preinvex functions and establish an estimate for the right-hand side of the HermiteHadamard operator inequality for such functions. Some Hermite-Hadamard type inequalities for the product of two operator preinvex functions is also given.

The following Theorem is a generalization of Theorem 1.3 for operator preinvex functions.

Theorem 3.1. Let $S \subseteq B(H)_{\text {sa }}$ be an invex set with respect to $\eta: S \times S \rightarrow$ $B(H)_{\text {sa }}$ and $\eta$ satisfies condition $C$. If for every $A, B \in S$ with spectra of $A, V:=$ $A+\eta(B, A)$ in the interval $I$, the function $f: I \rightarrow \mathbb{R}$ is operator preinvex with 
respect to $\eta$ for operators in $\eta$-path $P_{A V}$ and $k, p$ are positive integers, then the following inequalities holds

$$
\begin{gathered}
f\left(A+\frac{1}{2} \eta(B, A)\right) \leq \frac{1}{k^{p}} \sum_{i=0}^{k^{p}-1} f\left(A+\frac{2 i+1}{2 k^{p}} \eta(B, A)\right) \\
\leq \int_{0}^{1} f(A+t \eta(B, A)) d t \\
\leq \frac{1}{2 k^{p}} \sum_{i=0}^{k^{p}-1}\left[f\left(A+\frac{i+1}{k^{p}} \eta(B, A)\right)+f\left(A+\frac{i}{k^{p}} \eta(B, A)\right)\right] \\
\leq \frac{f(A)+f(B)}{2} .
\end{gathered}
$$

Proof. For $x \in H$ with $\|x\|=1$ and $t \in[0,1]$, we have

$$
\langle(A+t \eta(B, A)) x, x\rangle=(1-t)\langle A x, x\rangle+t\langle V x, x\rangle \in I,
$$

since $\langle A x, x\rangle \in S p(A) \subseteq I$ and $\langle V x, x\rangle \in S p(V) \subseteq I$.

Continuity of $f$ and (3.2) imply that the operator valued integral $\int_{0}^{1} f(A+$ $t \eta(B, A)) d t$ exists. Since $\eta$ satisfied condition $C$, by (2.2), for every $t \in[0,1]$ we have

$$
A+\frac{1}{2} \eta(B, A)=A+t \eta(B, A)+\frac{1}{2} \eta(A+(1-t) \eta(B, A), A+t \eta(B, A)) .
$$

Let $x \in H$ be a unit vector, define the real-valued function $\varphi:[0,1] \rightarrow \mathbb{R}$ given by $\varphi(t)=\langle f(A+t \eta(B, A)) x, x\rangle$. Since $f$ is operator preinvex, by the previous proposition $2.4, \varphi$ is a convex function on $[0,1]$. Utilizing the classical HermiteHadamard inequality for real-valued convex function $\varphi$ on the interval $\left[\frac{i}{k^{p}}, \frac{i+1}{k^{p}}\right]$, we get

$$
\varphi\left(\frac{2 i+1}{2 k^{p}}\right) \leq k^{p} \int_{\frac{i}{k^{p}}}^{\frac{i+1}{k^{p}}} \varphi(t) d t \leq \frac{\varphi\left(\frac{i}{k^{p}}\right)+\varphi\left(\frac{i+1}{k^{p}}\right)}{2}
$$

Summation of the above inequalities over $i=0, \cdots, k^{p}-1$ yields

$$
\sum_{i=0}^{k^{p}-1} \varphi\left(\frac{2 i+1}{2 k^{p}}\right) \leq k^{p} \int_{0}^{1} \varphi(t) d t \leq \sum_{i=0}^{k^{p}-1} \frac{\varphi\left(\frac{i}{k^{p}}\right)+\varphi\left(\frac{i+1}{k^{p}}\right)}{2} .
$$

Hence

$$
\begin{aligned}
& \frac{1}{k^{p}} \sum_{i=0}^{k^{p}-1} f(A\left.+\frac{2 i+1}{2 k^{p}} \eta(B, A)\right) \\
& \leq \int_{0}^{1} f(A+t \eta(B, A)) d t \\
& \leq \frac{1}{2 k^{p}} \sum_{i=0}^{k^{p}-1}\left[f\left(A+\frac{i+1}{k^{p}} \eta(B, A)\right)+f\left(A+\frac{i}{k^{p}} \eta(B, A)\right)\right] .
\end{aligned}
$$


Inequality (3.3) for $t=\frac{2 i+1}{k^{p}}$ and operator preinvexity $f$ imply that

$$
\begin{aligned}
& f\left(A+\frac{1}{2} \eta(B, A)\right) \\
& \quad \leq \frac{1}{2} f\left(A+\frac{2 i+1}{k^{p}} \eta(B, A)\right)+\frac{1}{2} f\left(A+\left(1-\frac{2 i+1}{k^{p}}\right) \eta(B, A)\right) .
\end{aligned}
$$

Summation of the above inequalities over $i=0, \cdots, k^{p}-1$ and the following equality

$$
\sum_{i=0}^{k^{p}-1} f\left(A+\frac{2 i+1}{k^{p}} \eta(B, A)\right)=\sum_{i=0}^{k^{p}-1} f\left(A+\left(1-\frac{2 i+1}{k^{p}}\right) \eta(B, A)\right)
$$

yield

$$
k^{p} f\left(A+\frac{1}{2} \eta(B, A)\right) \leq \sum_{i=0}^{k^{p}-1} f\left(A+\frac{2 i+1}{k^{p}} \eta(B, A)\right) .
$$

In the other hand, from preinvexity $f$ we have

$$
\begin{aligned}
& \frac{1}{2 k^{p}} \sum_{i=0}^{k^{p}-1}\left[f\left(A+\frac{i+1}{k^{p}} \eta(B, A)\right)+f\left(A+\frac{i}{k^{p}} \eta(B, A)\right)\right] \\
& \leq \frac{1}{2 k^{p}} \sum_{i=0}^{k^{p}-1}\left[\frac{i+1}{k^{p}} f(B)+\left(1-\frac{i+1}{k^{p}}\right) f(A)+\frac{i}{k^{p}} f(B)+\left(1-\frac{i}{k^{p}}\right) f(A)\right] \\
& \leq \frac{f(A)+f(B)}{2} .
\end{aligned}
$$

From inequalities (3.6), (3.8) and (3.9) we obtain (3.1).

A simple consequence of the above theorem is that the integral is closer to the left bound than to the right, namely we can state:

Corollary 3.2. With the assumptions in Theorem 3.1 we have the inequality

$$
\begin{aligned}
0 & \leq \int_{0}^{1} f(A+t \eta(B, A)) d t-f\left(A+\frac{1}{2} \eta(B, A)\right) \\
& \leq \frac{f(A)+f(B)}{2}-\int_{0}^{1} f(A+t \eta(B, A)) d t .
\end{aligned}
$$

Example 3.3. Let $S, f, \eta_{1}$ be as in Example 2.3, then we have

$$
\left(A+\frac{1}{2} \eta_{1}(B, A)\right)^{2} \leq \int_{0}^{1}\left(A+t \eta_{1}(B, A)\right)^{2} d t \leq \frac{A^{2}+B^{2}}{2}
$$

for every $A, B \in S$.

Let $S \subseteq B(H)_{s a}$ be an invex set with respect to $\eta: S \times S \rightarrow B(H)_{s a}$ and $f, g: I \rightarrow \mathbb{R}$ operator preinvex functions on the interval $I$ with respect to $\eta$ for operators in $\eta$-path $P_{A V}$. Then for every $A, B \in S$ with spectra of $A, V:=$ 
$A+\eta(B, A)$ in the interval $I$, we define real functions $M(A, B)$ and $N(A, B)$ on $H$ by

$$
\begin{aligned}
M(A, B)(x) & =\langle f(A) x, x\rangle\langle g(A) x, x\rangle+\langle f(B) x, x\rangle\langle g(B) x, x\rangle & & (x \in H), \\
N(A, B)(x) & =\langle f(A) x, x\rangle\langle g(B) x, x\rangle+\langle f(B) x, x\rangle\langle g(A) x, x\rangle & & (x \in H) .
\end{aligned}
$$

The following Theorem is a generalization of Theorem 1.1 for operator preinvex functions.

Theorem 3.4. Let $f, g: I \rightarrow \mathbb{R}^{+}$be operator preinvex functions on the interval $I$ with respect to $\eta$ and $\eta$ satisfies condition $C$. Then for any selfadjoint operators $A$ and $B$ on a Hilbert space $H$ with spectra $A, V$ in $I$, the inequality

$$
\begin{aligned}
\int_{0}^{1}\langle f(A+\operatorname{t\eta }(B, A)) x, x\rangle\langle g(A+\operatorname{t\eta }(B, A)) x, x\rangle d t & \\
& \leq \frac{1}{3} M(A, B)(x)+\frac{1}{6} N(A, B)(x),
\end{aligned}
$$

holds for any $x \in H$ with $\|x\|=1$.

Proof. Continuity of $f, g$ and (3.2) imply that the following operator valued integrals exist

$$
\int_{0}^{1} f(B+t \eta(B, A)) d t, \int_{0}^{1} g(A+t \eta(B, A)) d t, \int_{0}^{1}(f g)(A+t \eta(B, A)) d t .
$$

Since $f$ and $g$ are operator preinvex, therefore for $t$ in $[0,1]$ and $x \in H$ we have

$$
\begin{aligned}
& \langle f(A+\operatorname{t\eta }(B, A)) x, x\rangle \leq\langle(t f(B)+(1-t) f(A)) x, x\rangle, \\
& \langle g(A+\operatorname{t\eta }(B, A)) x, x\rangle \leq\langle(\operatorname{tg}(B)+(1-t) g(A)) x, x\rangle .
\end{aligned}
$$

From (3.11) and (3.12) we obtain

$$
\begin{aligned}
& \langle f(A+\operatorname{t\eta }(B, A)) x, x\rangle\langle g(A+\operatorname{t\eta }(B, A)) x, x\rangle \\
& \leq \quad(1-t)^{2}\langle f(A) x, x\rangle\langle g(A) x, x\rangle+t^{2}\langle f(B) x, x\rangle\langle g(B) x, x\rangle \\
& \quad+t(1-t)[\langle f(A) x, x\rangle\langle g(B) x, x\rangle+\langle f(B) x, x\rangle\langle g(A) x, x\rangle] .
\end{aligned}
$$

Integrating both sides of (3.13) over $[0,1]$ we get the required inequality (3.10).

Theorem 3.5. Let $f, g: I \rightarrow \mathbb{R}$ be operator preinvex functions on the interval $I$ with respect to $\eta$. If $\eta$ satisfies condition $C$, then for any selfadjoint operators $A$ and $B$ on a Hilbert space $H$ with spectra $A, V$ in $I$, the inequality 


$$
\begin{aligned}
\left\langle f\left(A+\frac{1}{2} \eta(B, A)\right) x, x\right\rangle\langle g(A+ & \left.\left.\frac{1}{2} \eta(B, A)\right) x, x\right\rangle \\
\leq & \frac{1}{2} \int_{0}^{1}\langle f(A+\operatorname{t\eta }(B, A)) x, x\rangle\langle g(A+t \eta(B, A)) x, x\rangle d t \\
& +\frac{1}{12} M(A, B)(x)+\frac{1}{6} N(A, B)(x),
\end{aligned}
$$

holds for any $x \in H$ with $\|x\|=1$.

Proof. Put $D=A+t \eta(B, A)$ and $E=A+(1-t) \eta(B, A)$, by (3.3) we have $A+\frac{1}{2} \eta(B, A)=D+\frac{1}{2} \eta(E, D)$. Since $f$ and $g$ are operator preinvex, therefore for any $t \in I$ and any $x \in H$ with $\|x\|=1$ we observe that

$$
\begin{gathered}
\left\langle f\left(A+\frac{1}{2} \eta(B, A)\right) x, x\right\rangle\left\langle g\left(A+\frac{1}{2} \eta(B, A)\right) x, x\right\rangle \\
=\left\langle f\left(D+\frac{1}{2} \eta(E, D)\right) x, x\right\rangle\left\langle g\left(D+\frac{1}{2} \eta(E, D)\right) x, x\right\rangle \\
\leq\left\langle\left(\frac{f(D)+f(E)}{2}\right) x, x\right\rangle\left\langle\left(\frac{g(D)+g(E)}{2}\right) x, x\right\rangle \\
\leq \frac{1}{4}[\langle f(D) x, x\rangle+\langle f(E) x, x\rangle][\langle g(D) x, x\rangle+\langle g(E) x, x\rangle] \\
\leq \frac{1}{4}[\langle f(D) x, x\rangle\langle g(D) x, x\rangle+\langle f(E) x, x\rangle\langle g(E) x, x\rangle] \\
+\frac{1}{4}[t\langle f(A) x, x\rangle+(1-t)\langle f(B) x, x\rangle][(1-t)\langle g(A) x, x\rangle+t\langle g(B) x, x\rangle] \\
+[(1-t)\langle f(A) x, x\rangle+t\langle f(B) x, x\rangle][t\langle g(A) x, x\rangle+(1-t)\langle g(B) x, x\rangle] \\
=\frac{1}{4}[\langle f(D) x, x\rangle\langle g(D) x, x\rangle+\langle f(E) x, x\rangle\langle g(E) x, x\rangle] \\
+\frac{1}{4} 2 t(1-t)[\langle f(A) x, x\rangle\langle g(A) x, x\rangle+\langle f(B) x, x\rangle\langle g(B) x, x\rangle] \\
+\left(t^{2}+(1-t)^{2}\right)[\langle f(A) x, x\rangle\langle g(B) x, x\rangle+\langle f(B) x, x\rangle\langle g(A) x, x\rangle] . \quad(3 .)
\end{gathered}
$$

We integrate both sides of (3.15) over [0,1] and obtain

$$
\begin{gathered}
\left\langle f\left(A+\frac{1}{2} \eta(B, A)\right) x, x\right\rangle\left\langle g\left(A+\frac{1}{2} \eta(B, A)\right) x, x\right\rangle \\
\leq \frac{1}{4} \int_{0}^{1}[\langle f(A+\operatorname{t\eta }(B, A)) x, x\rangle\langle g(A+\operatorname{t\eta }(B, A)) x, x\rangle \\
+\langle f(A+(1-t) \eta(B, A)) x, x\rangle\langle g(A+(1-t) \eta(B, A)) x, x\rangle] d t \\
+\frac{1}{12} M(A, B)(x)+\frac{1}{6} N(A, B)(x) .
\end{gathered}
$$

This implies the required inequality (3.14).

The following Theorem is a generalization of Theorem 3.1 in [2]. 
Theorem 3.6. Let the function $f: I \rightarrow \mathbb{R}^{+}$is continuous, $S \subseteq B(H)_{\text {sa }}$ be an open invex set with respect to $\eta: S \times S \rightarrow B(H)_{\text {sa }}$ and $\eta$ satisfies condition $C$. If for every $A, B \in S$ and $V=A+\eta(B, A)$ the function $f$ is operator preinvex with respect to $\eta$ on $\eta$-path $P_{A V}$ with spectra of $A$ and $V$ in $I$. Then, for every $a, b \in(0,1)$ with $a<b$ and every $x \in H$ with $\|x\|=1$ the following inequality holds,

$$
\begin{aligned}
\mid \frac{1}{2}\left\langle\int_{0}^{a} f(A+\right. & s \eta(B, A)) d s \quad x, x\rangle+\frac{1}{2}\left\langle\int_{0}^{b} f(A+s \eta(B, A)) d s x, x\right\rangle \\
& -\frac{1}{b-a} \int_{a}^{b}\left\langle\int_{0}^{t} f(A+s \eta(B, A)) d s x, x\right\rangle d t \mid \\
\leq & \frac{b-a}{8}\{\langle f(A+a \eta(B, A)) x, x\rangle+\langle f(A+b \eta(B, A)) x, x\rangle\} .
\end{aligned}
$$

Moreover we have

$$
\begin{gathered}
\| \frac{1}{2} \int_{0}^{a} f(A+s \eta(B, A)) d s+\frac{1}{2} \int_{0}^{b} f(A+s \eta(B, A)) d s \\
\quad-\frac{1}{b-a} \int_{a}^{b} \int_{0}^{t} f(A+s \eta(B, A)) d s d t \| \\
\leq \frac{b-a}{8}\|f(A+a \eta(B, A))+f(A+b \eta(B, A))\| \\
\leq \frac{b-a}{8}[\|f(A+a \eta(B, A))\|+\|f(A+b \eta(B, A))\|] .
\end{gathered}
$$

Proof. Let $A, B \in S$ and $a, b \in(0,1)$ with $a<b$. For $x \in H$ with $\|x\|=1$ we define the function $\varphi:[0,1] \rightarrow \mathbb{R}^{+}$by

$$
\varphi(t):=\left\langle\int_{0}^{t} f(A+s \eta(B, A)) d s x, x\right\rangle .
$$

Utilizing the continuity of the function $f$, the continuity property of the inner product and the properties of the integral of operator-valued functions we have

$$
\left\langle\int_{0}^{t} f(A+s \eta(B, A)) d s x, x\right\rangle=\int_{0}^{t}\langle f(A+s \eta(B, A)) x, x\rangle d s .
$$

Since $f(A+s \eta(B, A)) \geq 0$, therefore $\varphi(t) \geq 0$ for all $t \in I$. Obviously for every $t \in(0,1)$ we have

$$
\varphi^{\prime}(t)=\langle f(A+t \eta(B, A)) x, x\rangle \geq 0
$$

hence, $\left|\varphi^{\prime}(t)\right|=\varphi^{\prime}(t)$. Since $f$ is operator preinvex with respect to $\eta$ on $\eta$-path $P_{A V}$, by Proposition 2.4 the function $\varphi^{\prime}$ is convex. Applying Theorem 2.2 in [8] to the function $\varphi$ implies that

$$
\left|\frac{\varphi(a)+\varphi(b)}{2}-\frac{1}{b-a} \int_{a}^{b} \varphi(s) d s\right| \leq \frac{(b-a)\left(\varphi^{\prime}(a)+\varphi^{\prime}(b)\right)}{8},
$$

and we deduce that (3.16) holds. Taking supremum over both side of inequality (3.16) for all $x$ with $\|x\|=1$, we deduce that the inequality (3.17) holds. 


\section{REFERENCES}

1. T. Antczak, Mean value in invexity analysis, Nonlinear Analysis 60 (2005), 1471-1484.

2. A. Barani, A.G. Ghazanfari and S.S. Dragomir, Hermite-Hadamard inequality for functions whose derivatives absolute values are preinvex, J. Inequal. Appl. (2012), Article ID 247.

3. N.S. Barnett, P. Cerone and S.S. Dragomir, Some new inequalities for Hermite-Hadamard divergence in information theory, Stochastic analysis and applications. Vol. 3, 7-19, Nova Sci. Publ., Hauppauge, NY, 2003.

4. R. Bhatia, Matrix analysis, Springer-Verlag, New York, 1997.

5. C. Conde, A version of the Hermite-Hadamard inequality in a nonpositive curvature space, Banach J. Math. Anal. 6 (2012), no. 2, 159-167.

6. S.S. Dragomir, The Hermite-Hadamard type inequalities for operator convex functions, Appl. Math. Comput. 218 (2011), no. 3, 766-772.

7. S.S. Dragomir and C.E.M. Pearce, Selected Topics on Hermite-Hadamard Inequalities and applications, (RGMIA Monographs http:// rgmia.vu.edu.au/ monographs/ hermite hadamard.html), Victoria University, 2000.

8. S.S. Dragomir and R.P. Agarwal, Two inequalities for differentiable mappings and applications to special means of real numbers and to trapezoidal formula, Appl. Math. Lett. 11 (1998), no. 5, 91-95.

9. M.A. Hanson, On sufficiency of the Kuhn-Tucker conditions, J. Math. Anal. Appl. 80 (1981), 545-550.

10. E. Kikianty, Hermite-Hadamard inequality in the geometry of Banach spaces, PhD thesis thesis, Victoria University, 2010.

11. M. Klaričić Bakula and J. Pečarić, Note on some Hadamard-type inequalities, J. Inequal. Pure Appl. Math. 5 (2004), no. 3, Article 74.

12. S.R. Mohan and S.K. Neogy, On invex sets and preinvex function, J. Math. Anal. Appl. 189 (1995), 901-908.

13. M.S. Moslehian, Matrix Hermite-Hadamard type inequalities, Houston J. math 39 (2013) no $1,178-189$.

14. B.G. Pachpatte, On some inequalities for convex functions, RGMIA Res. Rep. Coll. (E) 6, (2003).

15. J. Pečarić, T. Furuta, J. Mićić Hot and Y. Seo, Mond-Pečarić Method in Operator Inequalities. Inequalities for Bounded Selfadjoint Operators on a Hilbert Space, Element, Zagreb, 2005.

16. M. Tunç, On some new inequalities for convex functions, Turkish J. Math. 36 (2012), $245-251$.

17. S. Wu, On the weighted generalization of the Hermite-Hadamard inequality and its applications, Rocky Mountain J. Math. 39 (2009), no. 5, 1741-1749.

18. X.M. Yang and D. Li, On properties of preinvex functions, J. Math. Anal. Appl. 256 (2001), 229-241.

Department of Mathematics, Lorestan University, P.O.Box 465, Khoramabad, IRAN.

E-mail address: ghazanfari.amir@gmail.com; ghazanfari.a@lu.ac.ir

E-mail address: alibarani2000@yahoo.com; barani.a@lu.ac.ir 